\title{
Height Adjustable Sine (HAS) Window for Impulse Response Modification of Signal Processing Systems
}

\author{
C. B. Mbachu
}

\begin{abstract}
A widow function, in signal processing and statistics, is a mathematical function that has zero values outside its chosen interval or limit of sequence, normally symmetric around the middle of the interval. Usually the middle of the window is either maximum or near maximum and tappers smoothly as it moves away to the sides. When another function or sequence of data is mathematically multiplied by the window function the product assumes the appearance and characteristics of the window function. That is, the product is also zero-valued outside the interval and tapering from middle to the sides. The impulse response data of some systems used in signal processing truncate suddenly when being designed. Window functions convert the sudden truncation of these impulse response data to gradual truncation. Without this gradual truncation the processing system will degrade the integrity of complex signals when they are applied to them; the degradation appears in form of signal distortions. Windows are used to weight these impulse response data so as to reduce the degradation and distortions. Several windows are in existence and they include Hamming, rectangular, Han, Kaiser, Triangular, Blackman, Sine, Blackman-Harris, Gaussian, Doph-Chebyshev, and Lanczos, windows. Others are Parzen, Nuttall, flat top, and Turkey, windows. The characteristics of the signal to be processed, types of systems to be implemented and quality of outputs desired determine the best window to weight any particular impulse response data. In this work a new window referred to as height adjustable sine (HAS) window is developed to join the list of the existing windows. The amplitude or height of the new window is varied by varying a parameter called amplitude adjustment parameter. The quality of the window is investigated by analyzing its amplitude, magnitude and phase responses in frequency domain. Result shows that the window is stable and linear.
\end{abstract}

Index Terms-HAS, Windows, Responses in Frequency Domain.

\section{INTRODUCTION}

A digital processing system such as a filter is designed to implement an algorithm that converts an input sequence $\mathrm{x}(\mathrm{n})$ into a desired output sequence $y(n)$ [1]. Some of the systems can perform the functions of signal separation in which case noises, interferences or unwanted signals are removed from the desired signal, and signal restoration in which case a distorted signal can be restored to its quality form [2]. Fourier series method is an effective mathematical tool in the design of some processing systems. In some practical situations, the impulse response data resulting from the design are of infinite duration [1], [3]-[6]. The most straight forward approach to convert this infinite duration response

Published on March 27, 2020.

Mbachu C. B. is with Chukwuemeka Odumegwu Ojukwu University, Uli, Anambra State, Nigeria (e-mail: dambac614@gmail.com) to finite duration response is by truncation of the infinite duration impulse sequence. This truncation incidentally results to oscillations at the point of discontinuities for a processing system designed from it. The oscillations manifest as ripples in the magnitude response of the system and may cause distortion of the input signal to the system. In applications where these oscillations cannot be tolerated window functions are used to weight the systems. This windowing reduces the sudden truncation of the system to gradual truncation and hence overcoming the oscillations [1], [3]-[7]. Many windows are already applicable, but in this research a new window titled height adjustable sine (HAS) window is developed and its suitability in designing signal processing systems investigated.

\section{EXISTING WINDOW FUNCTIONS}

With $\mathrm{M}$ as the window length, the mathematical models of some windows are as presented below.

\section{A. Triangular (Bartlett) Window}

This is as obtained from [2]-[4],

$w(n)=\left\{\begin{array}{l}2 n /(M-1), 0 \leq n \leq(M-1) / 2 \\ 2-[2 n /(M-1)], \frac{M-1}{2} \leq n \leq M-1\end{array}\right\}$

\section{B. Rectangular Window}

As in [4], [8],

$w(n)=1,0 \leq n \leq M-1$

\section{Hanning Window}

As in [4], [14], [15],

$$
w(n)=0.5-0.5 \cos \frac{2 \pi n}{M-1}, 0 \leq n \leq M-1
$$

\section{Hamming Window}

From [4], [14], [15],

$$
w(n)=0.54-0.46 \cos \frac{2 \pi n}{M-1}, 0 \leq n \leq M-1
$$

Where $0 \leq n \leq M-1$

\section{E. Blackman Window}

As in [4], [9], [14],

$$
w(n)=0.42+0.5 \cos \frac{2 \pi n}{M-1}+0.08 \cos \frac{4 \pi n}{M-1}
$$


Where $0 \leq n \leq M-1$

\section{F. Blackman-Harris Window}

From [9], [10], [16], [17]

$$
\begin{aligned}
w(n)=a_{0} & +a_{1} \cos \frac{2 \pi n}{M-1}+a_{2} \cos \frac{4 \pi n}{M-1} \\
& +a_{3} \cos \frac{6 \pi n}{M-1}
\end{aligned}
$$

Where, $-\frac{M-1}{2} \leq n \leq \frac{M-1}{2}$, and $\mathrm{a}_{0}=0.35875, \mathrm{a}_{1}=-0.48829, \mathrm{a}_{2}$ $=0.14128, \mathrm{a}_{3}=-0.01168$

\section{G. Gaussian Window}

From [9], [10], [11],

$$
w(n)=e^{-\frac{1}{2}}\left[\alpha \frac{n}{N / 2}\right]^{2}
$$

Where, $\quad-\frac{M-1}{2} \leq n \leq \frac{M-1}{2} \quad$ and $\alpha \geq 2$. The term $\alpha$ is the reciprocal of the standard deviation. The width of the window is inversely related to the value of $\alpha$; the larger the $\alpha$, the narrower the window.

\section{H. Kaiser Window}

From [1], [9], [12], [13], [18],

$$
\begin{gathered}
W_{k}(\beta, n)=\frac{J_{o}\left[\beta\left[1-\left(\frac{2 n}{M-1}\right)^{2}\right]^{1 / 2}\right]}{J_{o} \beta} \\
\text { Where }-\frac{M-1}{2} \leq n \leq \frac{M-1}{2} \\
\text { Where } J_{o}(P)=1+\sum_{k=0}^{\infty}\left[(P / 2)^{k} / k !\right]^{2}
\end{gathered}
$$

$\mathrm{J}_{\mathrm{o}}(\mathrm{x})$ is the modified Bessel function of the first kind of order zero.

\section{Sine Window}

According to [19], sine window function is given as in (10):

$$
w(n)=\sin \frac{180 n}{M-1}, 0 \leq n \leq M-1
$$

\section{J. Nuttall Window}

As can be seen from [20], [21], the window is depicted as (11)

$$
\begin{aligned}
& w(n)=a_{0}-a_{1} \cos \frac{2 \pi n}{M-1}+a_{2} \cos \frac{4 \pi n}{M-1} \\
& -a_{3} \cos \frac{6 \pi n}{M-1}
\end{aligned}
$$

Where $-\frac{M-1}{2} \leq n \leq \frac{M-1}{2} \quad \mathrm{a}_{0}=0.355768, \mathrm{a}_{1}=0.487396, \mathrm{a}_{2}=$ $0.144232, \mathrm{a}_{3}=0.012604$

\section{DEVELOPMENT OF HAS WINDOW FUNCTION}

The diagram of the height adjustable sine window function is shown as Fig. 1 below.

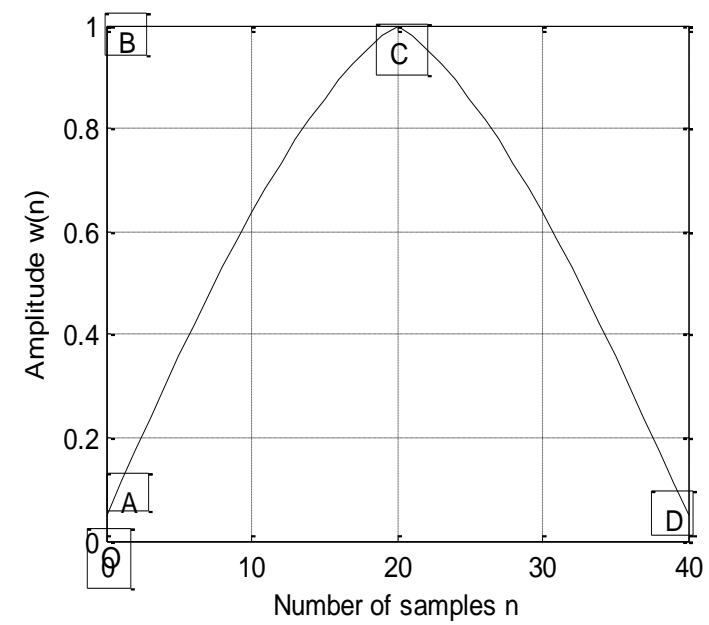

Fig. 1. Height Adjustable Sine Window Function

From Fig.1 which is a unit function, the straight line distance $\mathrm{OB}=1$. Also the straight line distance $\mathrm{OA}=\alpha$, where $\alpha$ is the height or amplitude adjustment parameter and can vary from 0 to 1 to adjust the function amplitude or height. Therefore, the height or amplitude of the function is

$$
\mathrm{AB}=(1-\alpha)
$$

Let $\mathrm{AD}=\mathrm{L}$ and $\mathrm{L}=\mathrm{M}-1$, where $\mathrm{M}$ is the length of the window, starting from the origin ' 0 ' and $\mathrm{L}$ is the order of the window.

$$
(\mathrm{AD}) / 2=\mathrm{L} / 2
$$

Let the angle made by AC curve of the function be $\varphi$. At $\mathrm{C}$, $\varphi=\theta$

$$
\begin{aligned}
\sin \theta & =(1-\alpha) \\
\theta & =\sin ^{-1}(1-\alpha)
\end{aligned}
$$

Also at $\mathrm{C}$ the corresponding value of $\mathrm{AD}$ along the horizontal axis is (L/2) from (13).

$$
\begin{gathered}
\varphi \cdot \frac{L}{2}=\theta=\sin ^{-1}(1-\alpha) \\
\varphi=\frac{2 \sin ^{-1}(1-\alpha)}{L}
\end{gathered}
$$

For the rising side $\mathrm{AC}$, the function is

$$
\begin{aligned}
& w_{1}(n)=\alpha+\sin (\varphi n) \\
= & \alpha+\sin \left[\frac{2 \sin ^{-1}(1-\alpha)}{L} n\right], 0 \leq n \leq \frac{M-1}{2}
\end{aligned}
$$

Where $\mathrm{n}$ is the instantaneous window length as shown in Fig. 1. The falling side CD of the function is the same as the rising side $\mathrm{AC}$ except that it is the obtuse angle side whereas $\mathrm{AC}$ is the acute angle side. Recall that for acute angle, $\theta$ $\sin \theta=\sin (180-\Theta)$ 
Therefore,

$$
\sin (\varphi n)=\sin [(L-n) \varphi]
$$

Where, $\varphi$ is an acute angle.

By this reasoning, for the $\mathrm{CD}$ curve

$$
\begin{aligned}
& w_{2}(n)=\alpha+\sin [(L-n) \varphi] \\
= & \alpha+\sin \left[\frac{2 \sin ^{-1}(1-\alpha)}{L}(L-n)\right], \frac{M-1}{2} \leq n \leq M-1
\end{aligned}
$$

The height adjustable sine window function $\mathrm{w}(\mathrm{n})$ is obtained by combining (18) and (20):

$$
w(n)=\left[\begin{array}{l}
\alpha+\sin \left[\frac{2 \sin ^{-1}(1-\alpha)}{L} n\right], 0 \leq n \leq \frac{M-1}{2} \\
\alpha+\sin \left[\frac{2 \sin ^{-1}(1-\alpha)}{L}(L-n)\right], \frac{M-1}{2} \leq n \leq M-1
\end{array}\right]
$$

Equation (21) is the developed new HAS window function. As the amplitude adjustment parameter $\alpha$ varies from " 0 " to " 1 ," the function varies from sine window to rectangular window. At the value of $\alpha=0$ it is a perfect sine window function whereas at the value of $\alpha=1$ it is a perfect rectangular window function.

\section{FREQUENCY CHARACTERISTICS OF HAS WINDOW FUNCTION}

The usefulness of HAS window function can be determined by analyzing its behavior in frequency domain. Such behavior includes amplitude response in frequency domain, magnitude response in frequency domain and phase response in frequency domain. The window adjustment parameter $\alpha$ varies the amplitude or height of the window for a fixed window length. This amplitude variation correspondingly varies the main and side lobes of the magnitude response of the function and can also affect the linearity of the phase of the function. The effects of $\alpha$ on the responses can be examined by considering six different values of it $(0.0,0.01,0.02,0.03,0.04,0.05)$ when the length of the window is $M=31$. Practically the value of $\alpha$ depends on the type and frequency of signal to be processed, interference level and desired output and the length of the window, while the value of $\mathrm{M}$ is the same as the number of elements in the impulse response sequence of the processing system being designed. The amplitude, magnitude and phase responses of the function in frequency domain for various values of $\alpha$ are depicted below.

\section{A. Responses when $\alpha=0.00$}

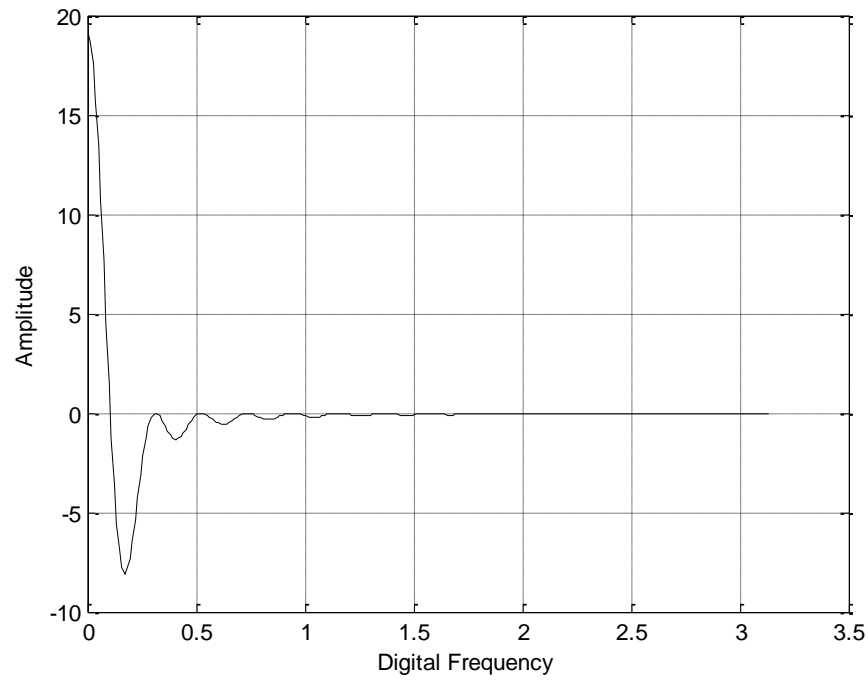

Fig. 2. Amplitude Response in Frequency Domain when $\alpha=0.00$

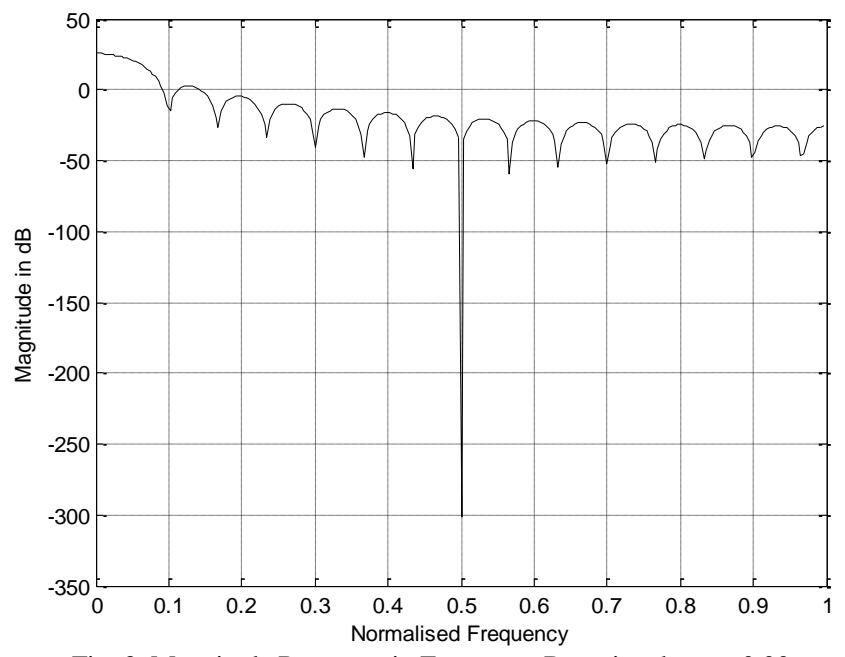

Fig. 3. Magnitude Response in Frequency Domain when $\alpha=0.00$

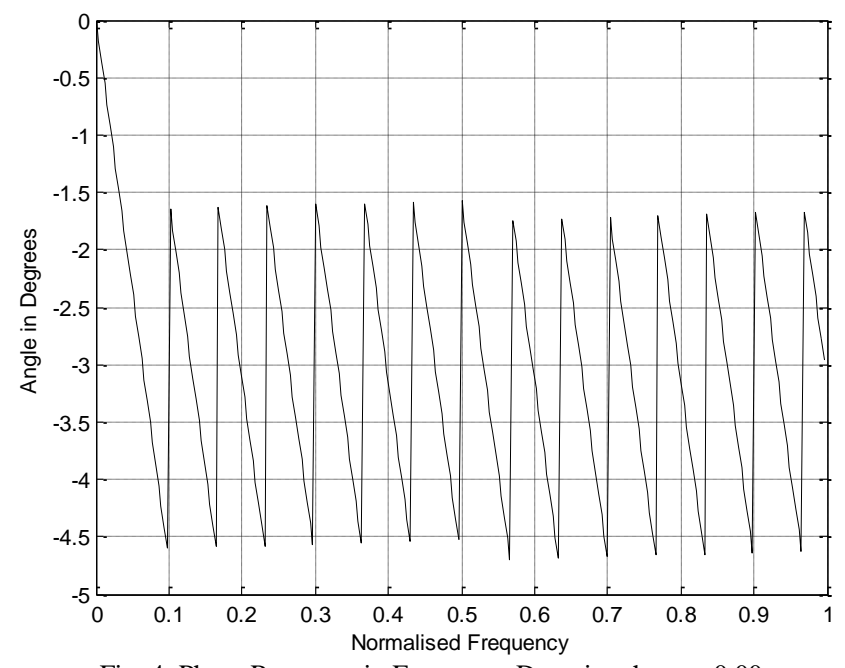

Fig. 4. Phase Response in Frequency Domain when $\alpha=0.00$

\section{B. Responses when $\alpha=0.01$}




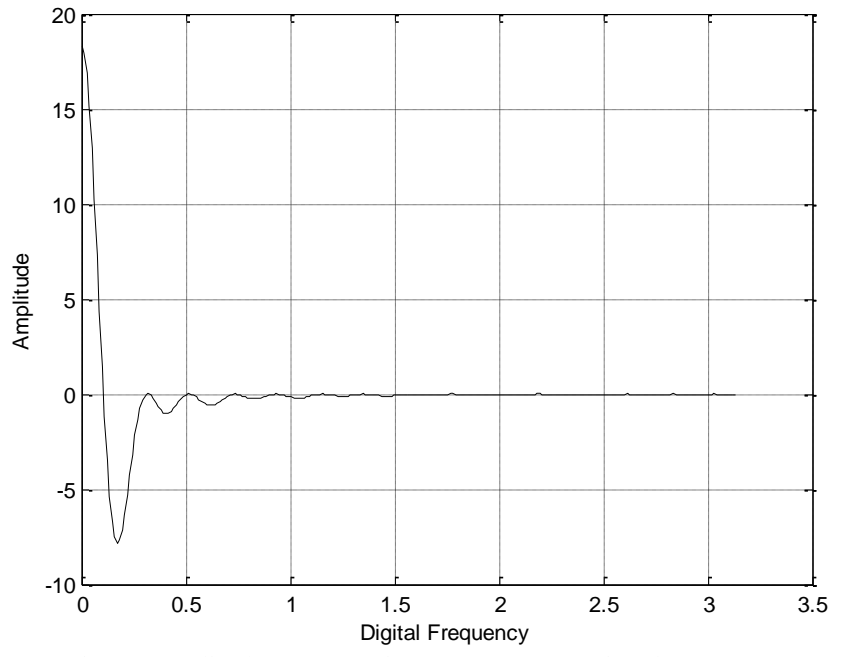

Fig. 5. Amplitude Response in Frequency Domain when $\alpha=0.01$

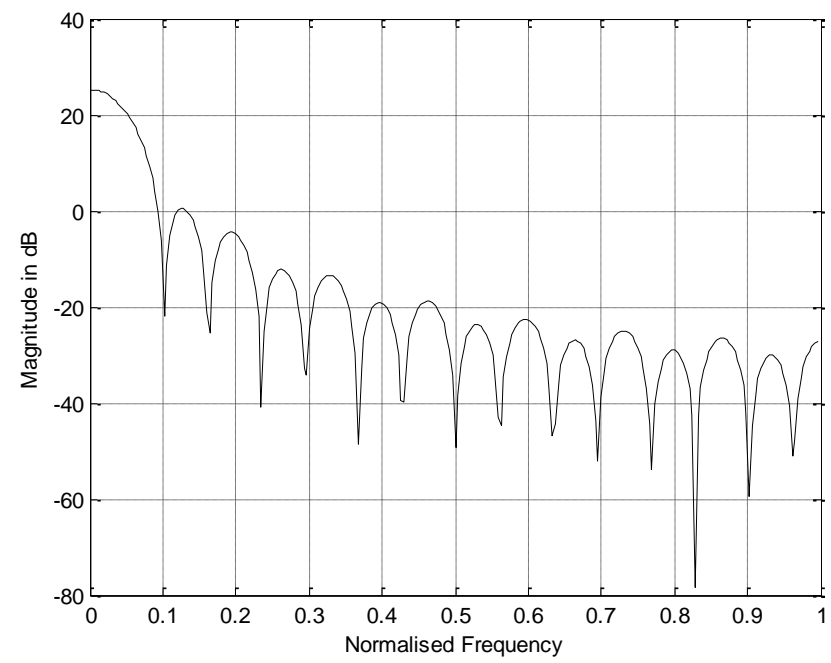

Fig. 6. Magnitude Response in Frequency Domain when $\alpha=0.01$

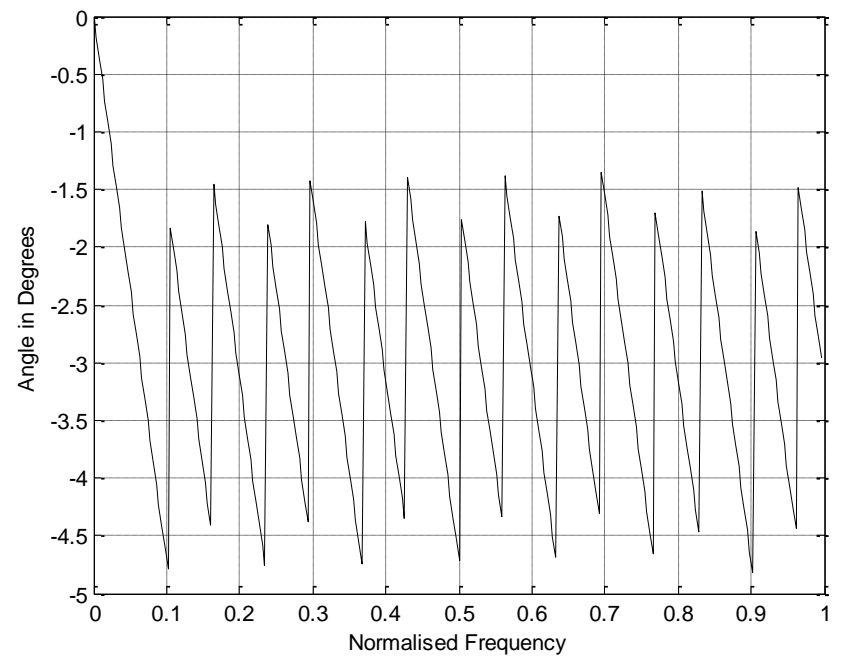

Fig. 7. Phase Response in Frequency Domain when $\alpha=0.01$

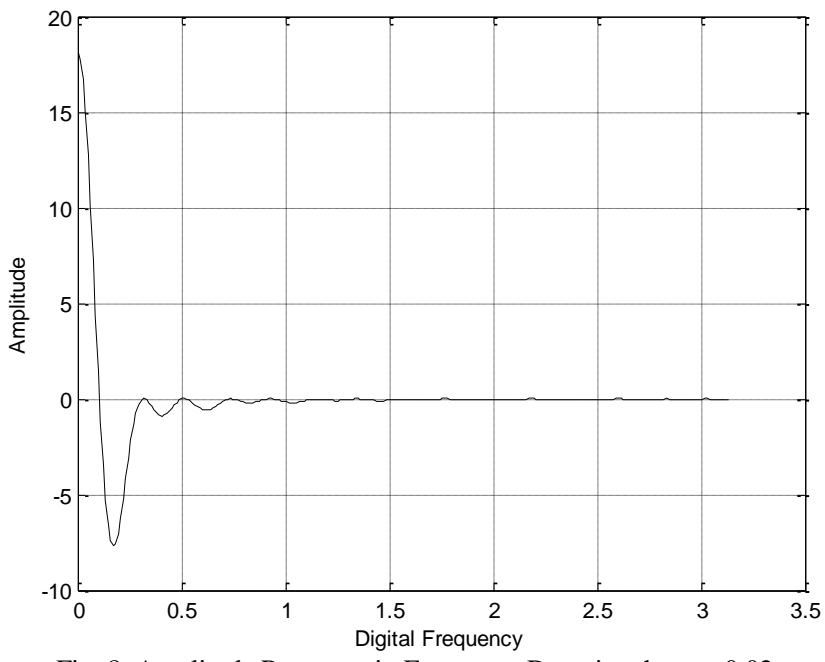

Fig. 8. Amplitude Response in Frequency Domain when $\alpha=0.02$

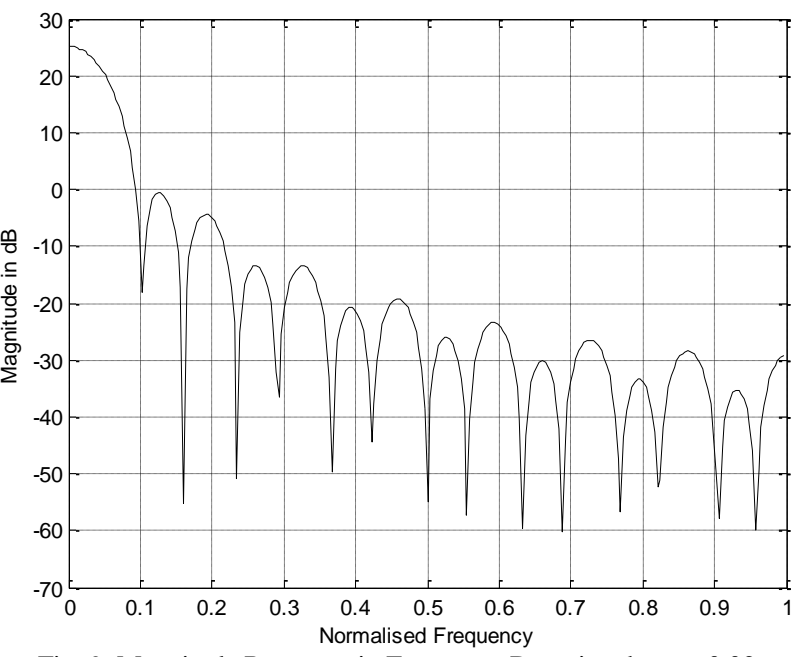

Fig. 9. Magnitude Response in Frequency Domain when $\alpha=0.02$

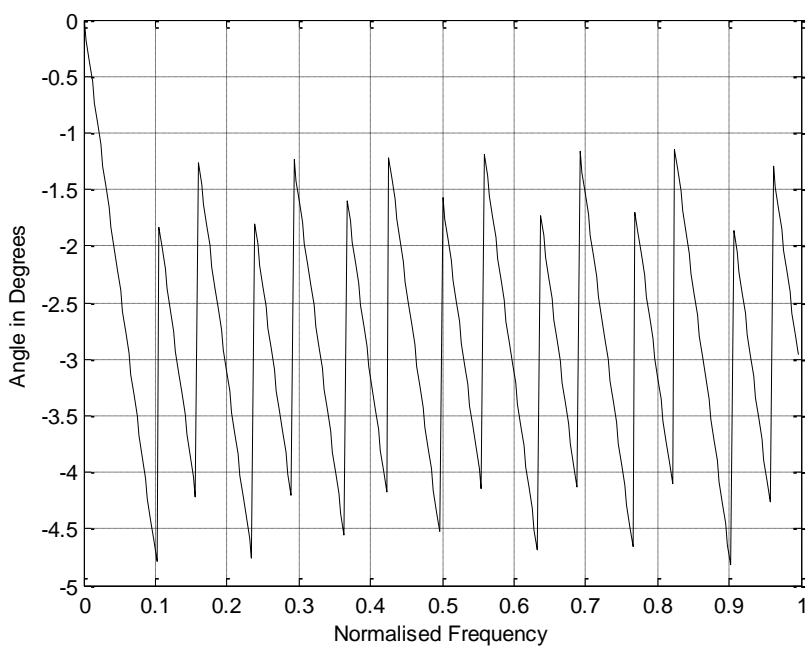

Fig. 10. Phase Response in Frequency Domain when $\alpha=0.02$ 


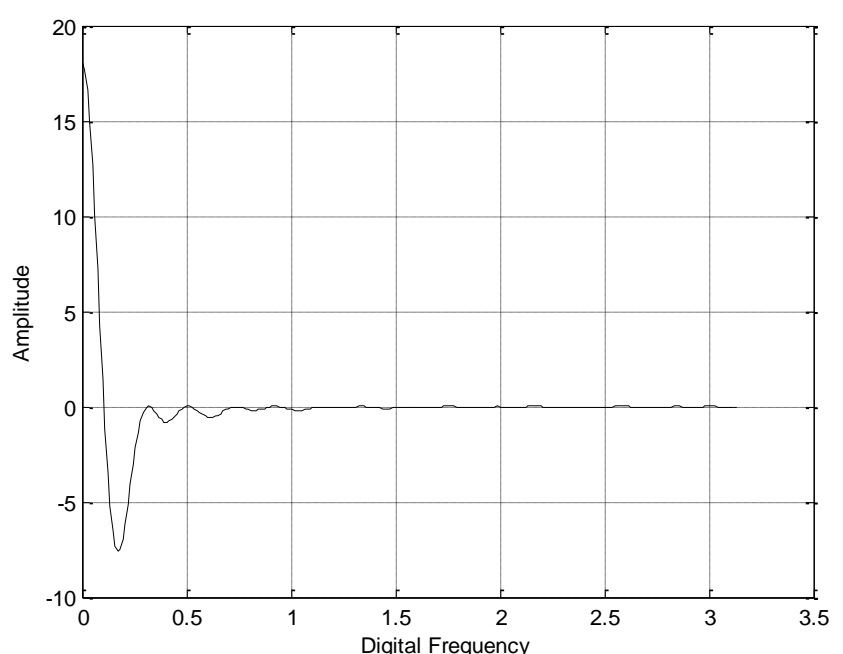

Fig. 11. Amplitude Response in Frequency Domain when $\alpha=0.03$

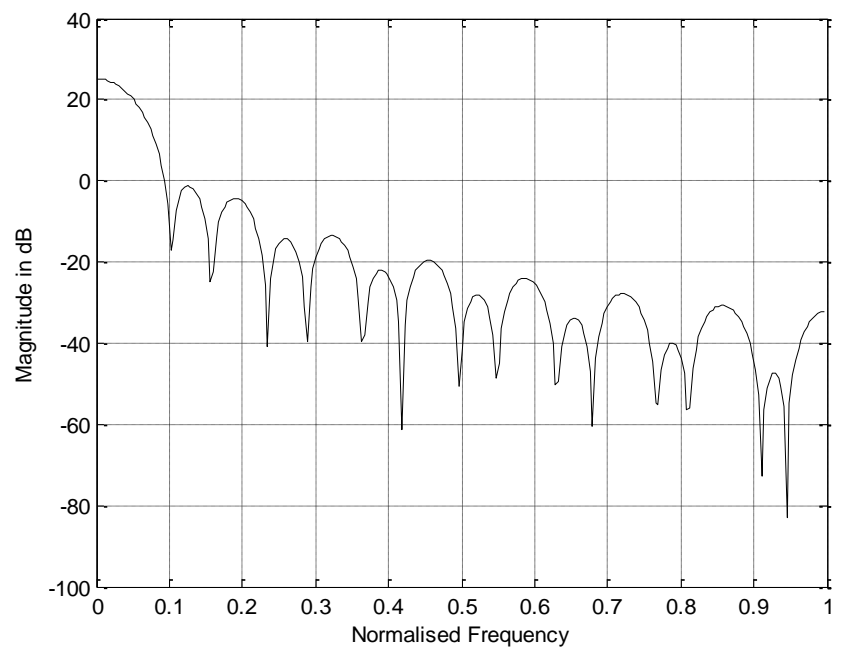

Fig. 12. Magnitude Response in Frequency Domain when $\alpha=0.03$

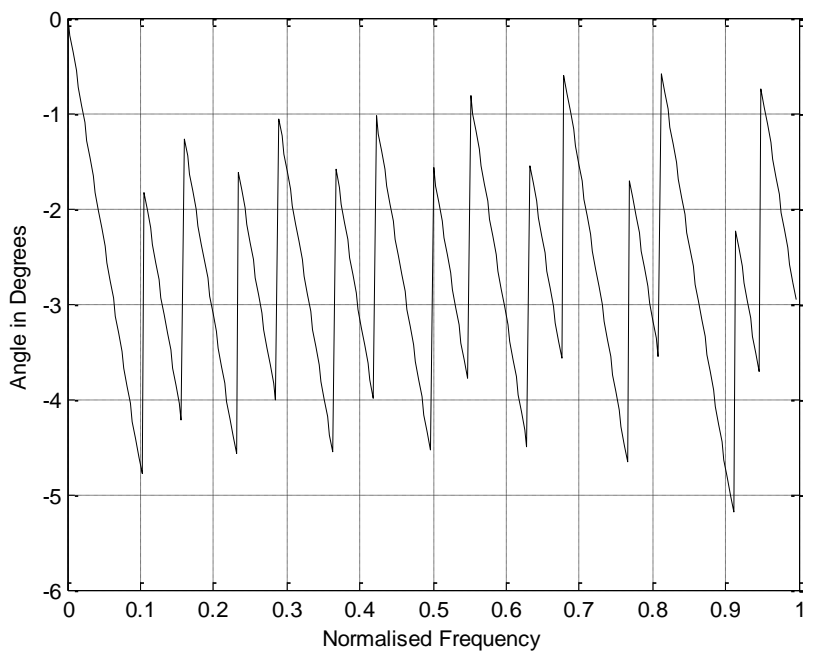

Fig. 13. Phase Response in Frequency Domain when $\alpha=0.03$

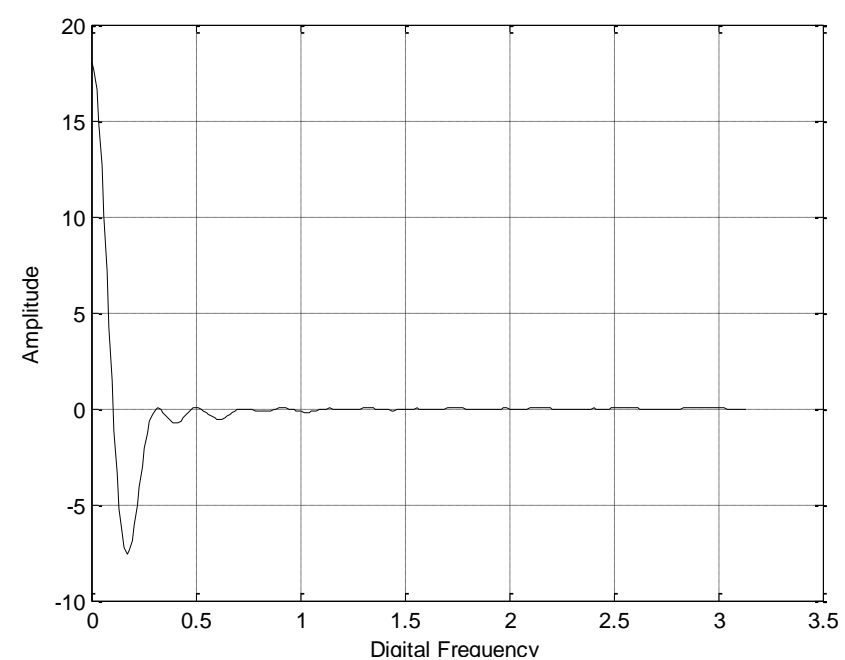

Fig. 14. Amplitude Response in Frequency Domain when $\alpha=0.04$

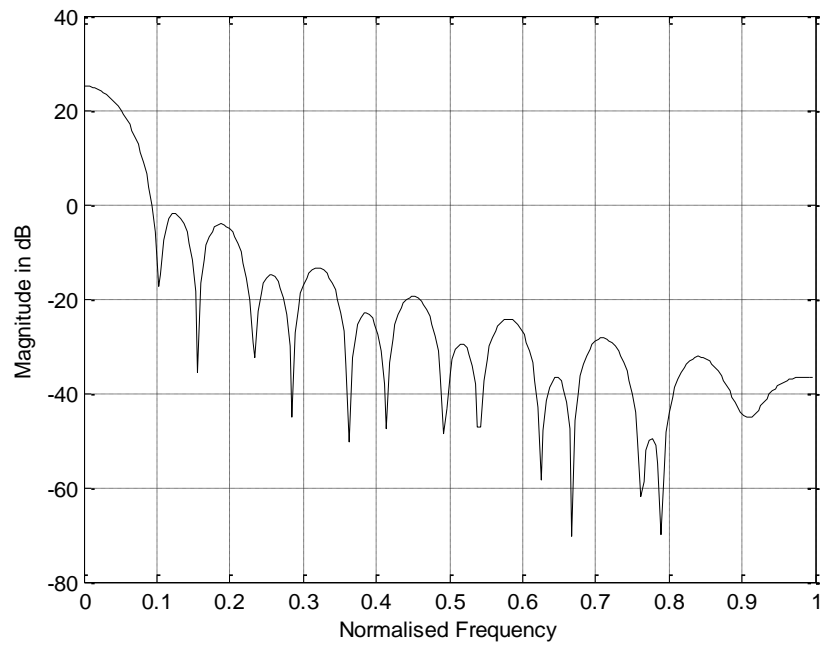

Fig. 15. Magnitude Response in Frequency Domain when $\alpha=0.04$

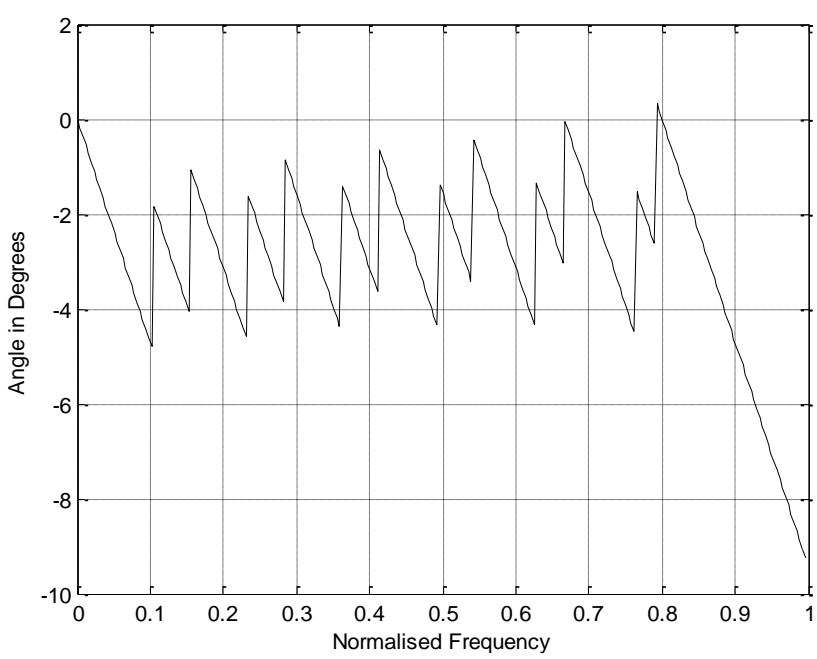

Fig. 16. Phase Response in Frequency Domain when $\alpha=0.04$

E. Responses when $\alpha=0.04$

\section{F. Responses when $\alpha=0.05$}




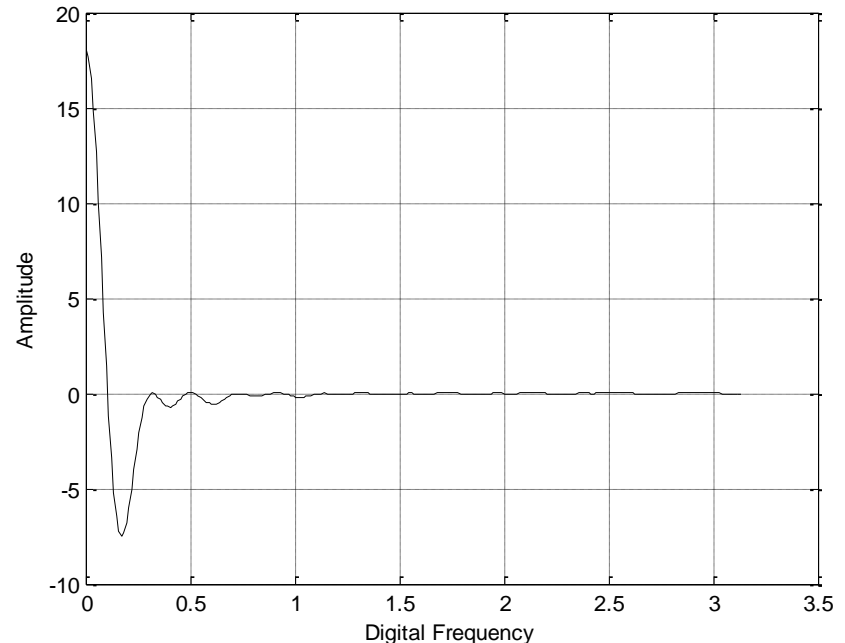

Fig. 17. Amplitude Response in Frequency Domain when $\alpha=0.05$

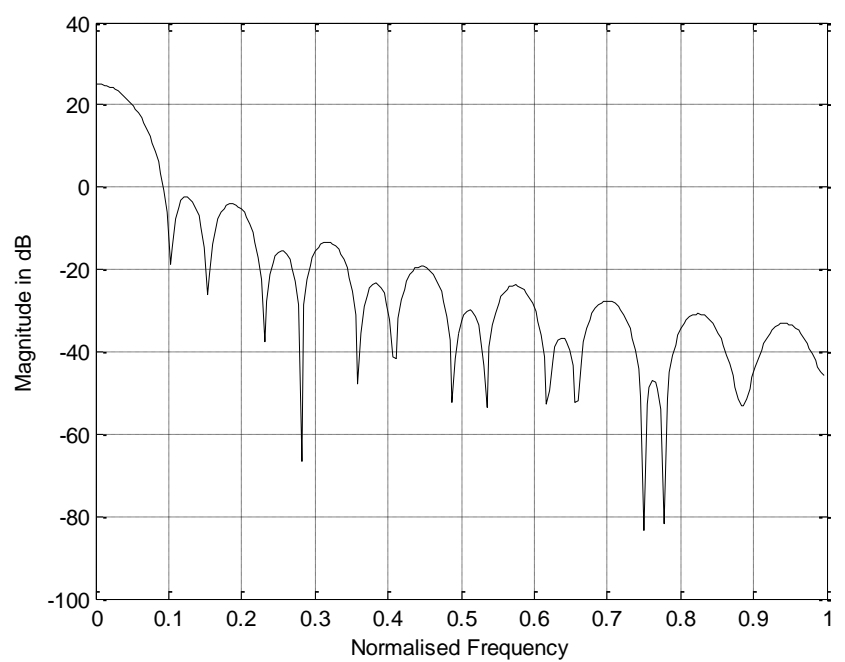

Fig. 18. Magnitude Response in Frequency Domain when $\alpha=0.05$

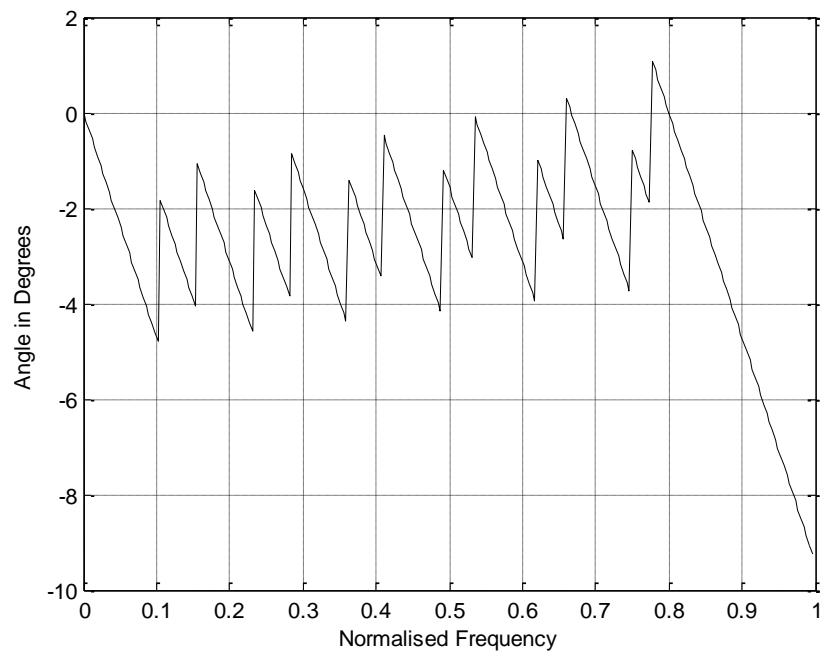

Fig. 19. Phase Response in Frequency Domain when $\alpha=0.05$

The amplitude responses of the function in frequency domain as shown above are indicating stability because they gradually reduce as the frequency increases and eventually collapses to zero. The magnitude responses in frequency domain as shown are also indicating good stability because the lobes continue to dwindle as the frequency increases and no sustained oscillation is observed. The stability increases as the values of $\alpha$ increases. The phase responses in frequency domain show non linearity for lower values of $\alpha$ but the nonlinearity started improving from $\alpha=0.4$, though no value of $\alpha$ gave very linear phase response.

It is also necessary to point out that the value of $\alpha$ depends on the window length. This is demonstrated from Fig. 20 to Fig. 31 where the window length is increased to 40 , giving rise to relative linearity of phase manifesting from 0.03 and above.

G. Responses when Window Length is 40

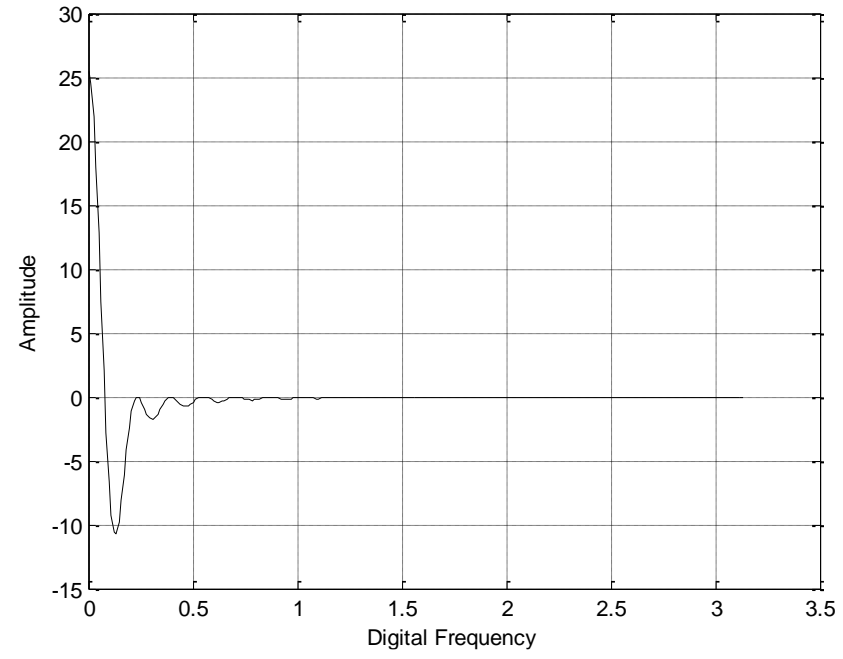

Fig. 20. Amplitude Response when Window Length is 40 and $\alpha=0.00$

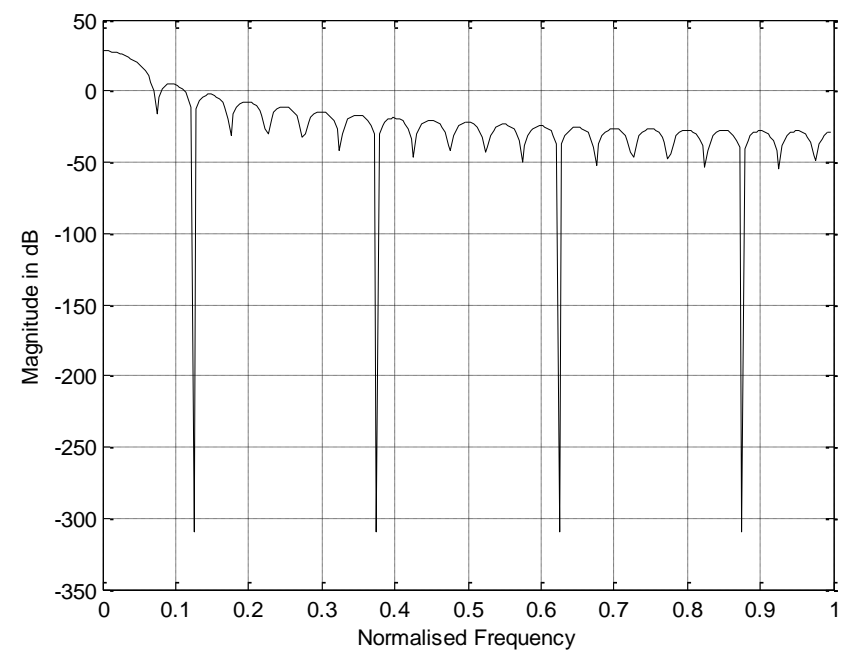

Fig. 21. Magnitude Response when Window Length is 40 and $\alpha=0.00$

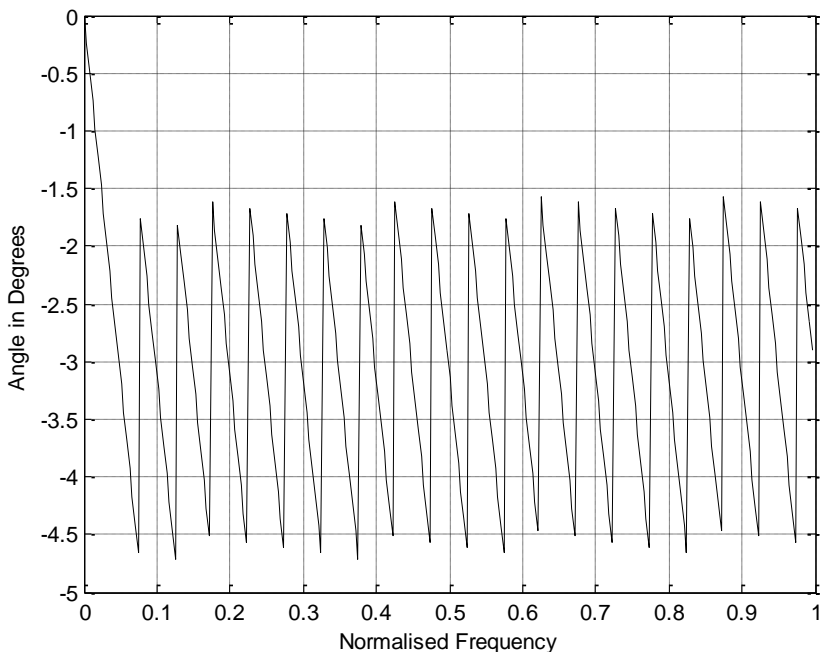

Fig. 22. Phase Response when Window Length is 40 and $\alpha=0.00$ 


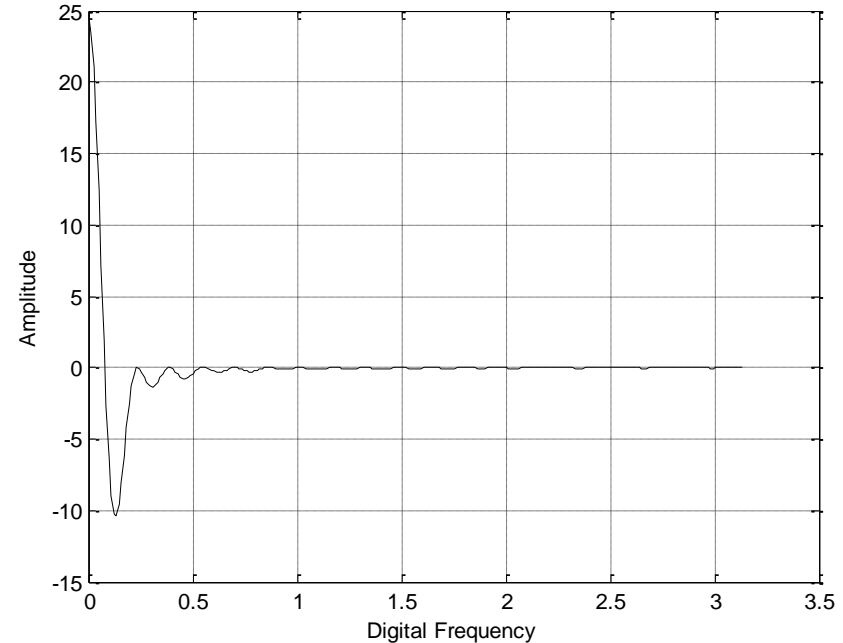

Fig. 23. Amplitude Response when Window Length is 40 and $\alpha=0.01$

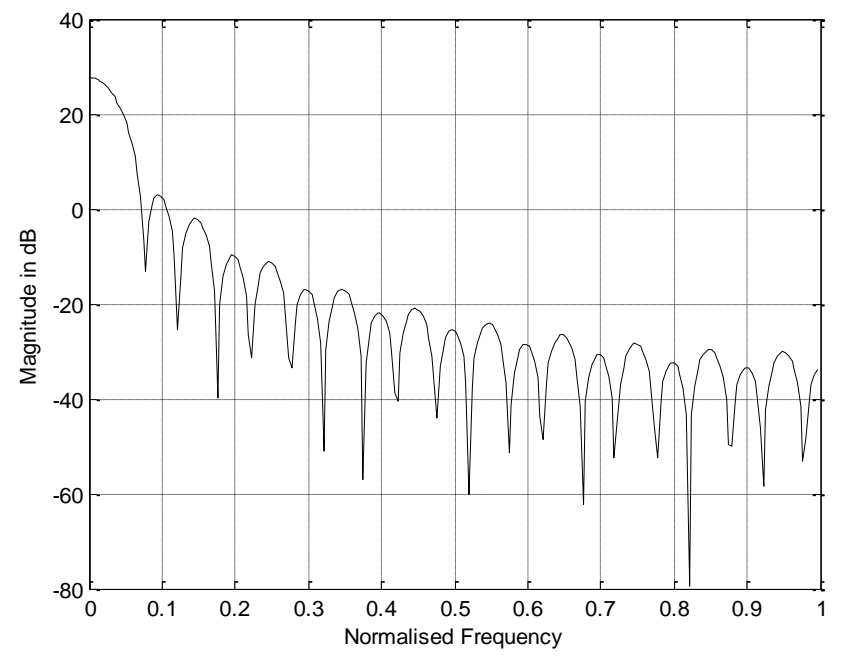

Fig. 24. Magnitude Response when Window Length is 40 and $\alpha=0.01$

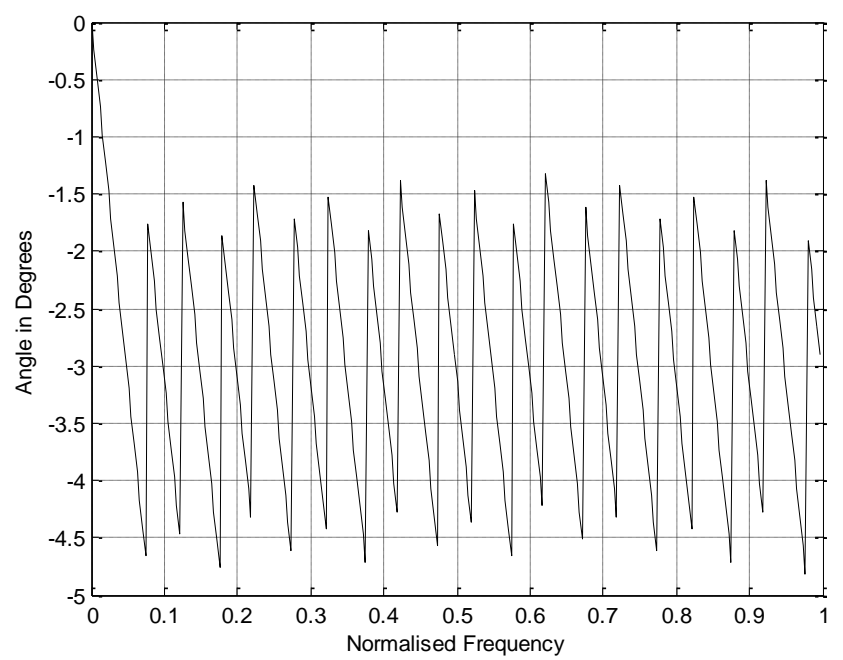

Fig. 25. Phase Response when Window Length is 40 and $\alpha=0.01$

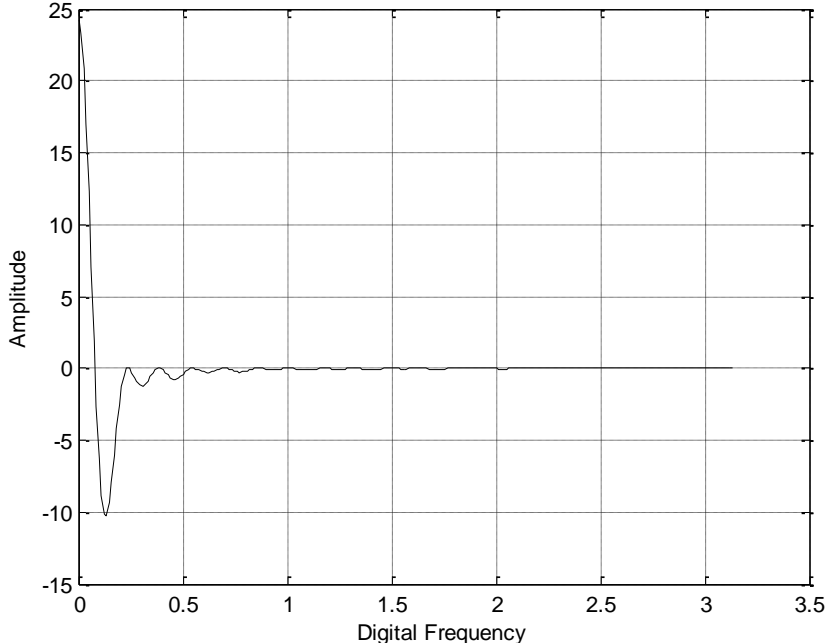

Fig. 26. Amplitude Response when Window Length is 40 and $\alpha=0.02$

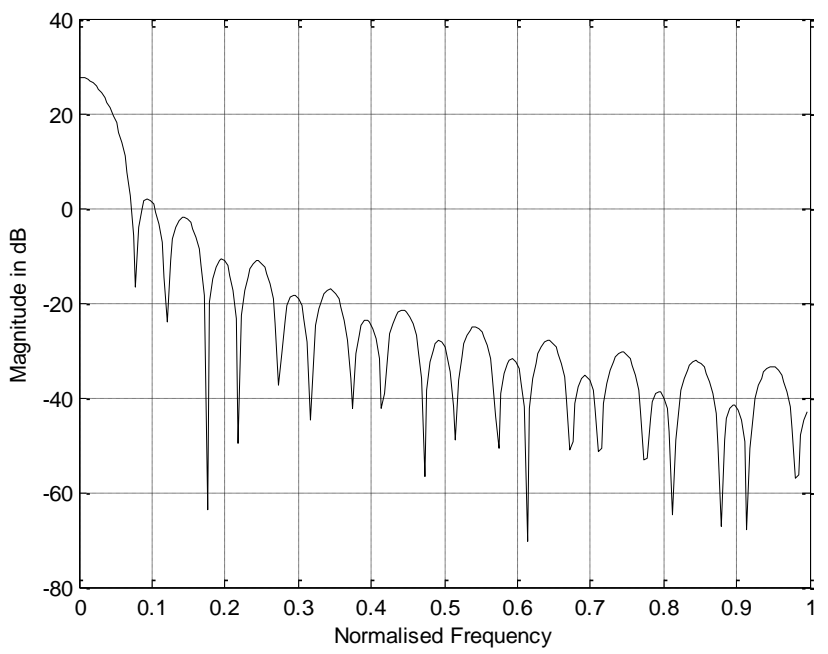

Fig. 27. Magnitude Response when Window Length is 40 and $\alpha=0.02$

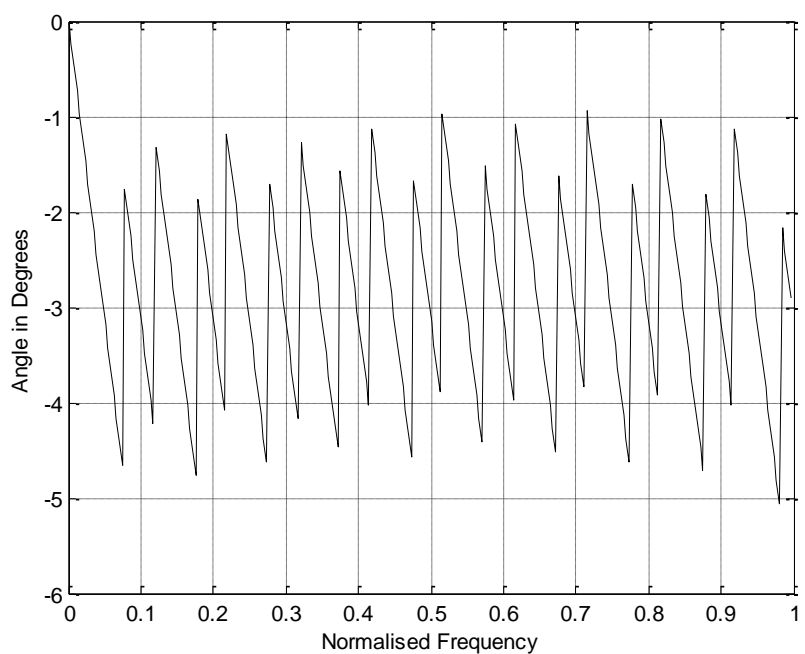

Fig. 28. Phase Response when Window Length is 40 and $\alpha=0.02$ 


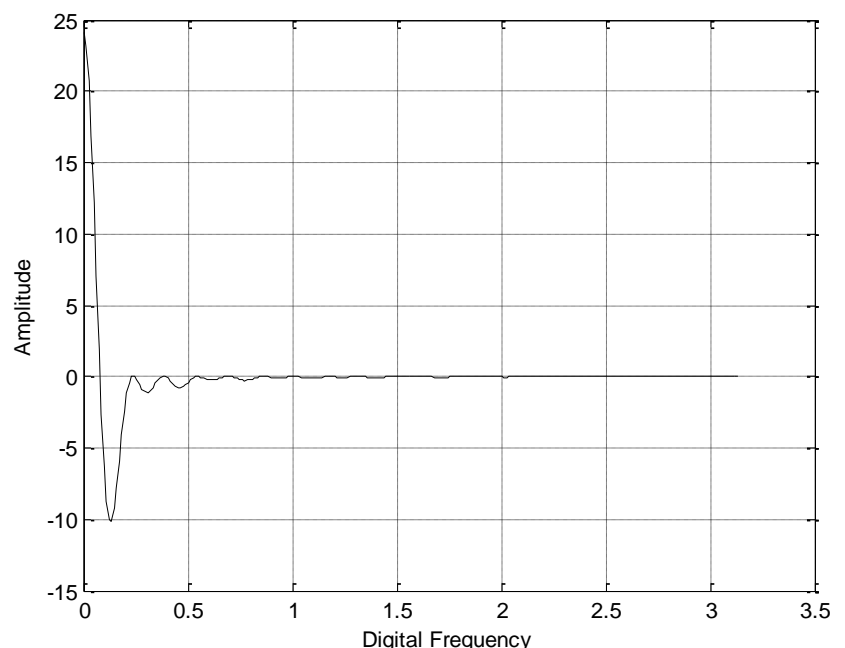

Fig. 29. Amplitude Response when Window Length is 40 and $\alpha=0.03$

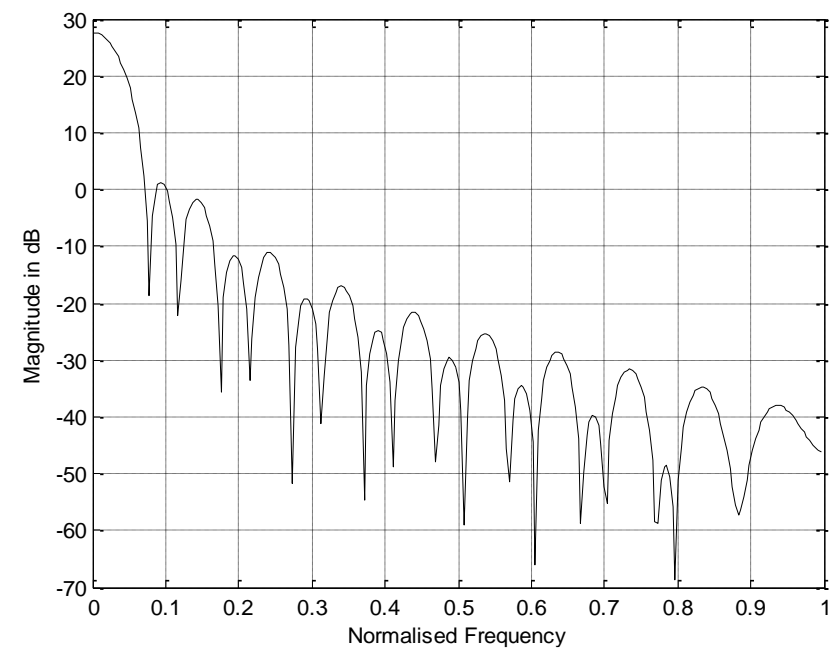

Fig. 30. Magnitude Response when Window Length is 40 and $\alpha=0.03$

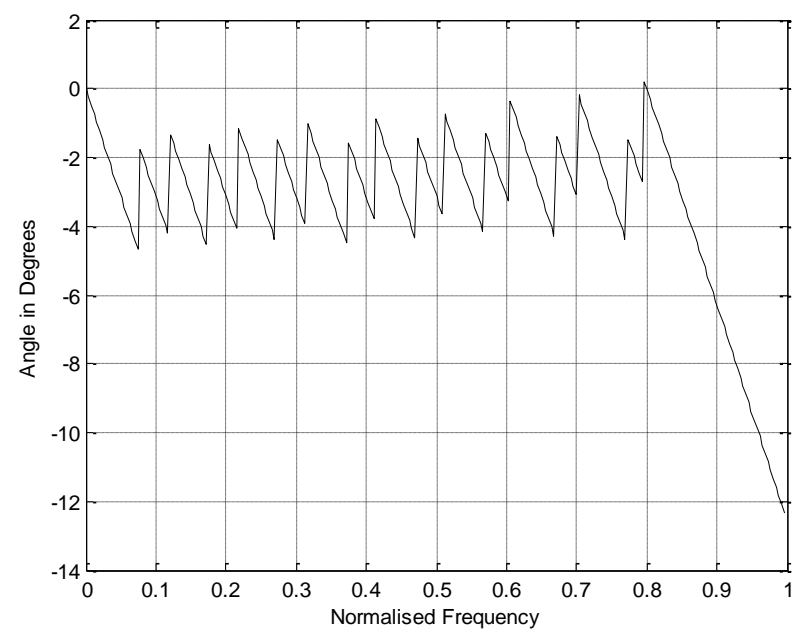

Fig. 31. Phase Response when Window Length is 40 and $\alpha=0.03$

\section{CONCLUSION}

From the characteristics of HAS window function it can be deduced that the function can be used in designing signal processing systems within its linear range to eliminate Gibbs phenomenon without compromising the integrity of the system. The degree of stability and linearity depends on the amplitude adjustment parameter with respect to the length of the window. For example, for a window length of 31 the phase response shows some degree of linearity up to the amplitude adjustment parameter of 0.03 , whereas for a window length of 40 the phase response is somehow linear only up to the amplitude adjustment parameter of 0.02 . The implication therefore is that HAS window can only be used on such signal processing systems that have lengths within the good performance range of lengths of the window if the integrity of the signal to be processed is to be maintained.

\section{REFERENCES}

[1] N. Sarkar, Elements of Digital Signal Processing, Delhi, India: Khanna Publishers, 2003.

[2] S. W. Smith, The Scientist's and Engineer's Guide to Digital Signal Processing, California: Technical Publishing, 2001.

[3] T. J. Cavicchi, Digital Signal Processing,New York: John Wiley and Sons, 2000.

[4] S. K Mitra, Digital Signal Processing, A Computer - Based Approach: Mc Graw Hill, 2001.

[5] K. D. Pranab, H. He-Sung, and K. Jon-Myon, "Design and Implementation of Digital Filters for Audio Signal Processing," Third International Forum on Strategic Technologies, pp. 332 - 335, 2008.

[6] W. Putman and J. Smith, "Design of Fractional Delay Filters using Convex Optimization," Workshop on Applications of Signal Processing to Audio and Acoustrics, 1997

[7] K. D. Pranab, C. Ui-Pil, and K. Jon-Myon, "Implementation and Performance Analysis of Real Time Digital Filter for Audio Signals," Third International Conference on Strategic Technologies, pp. 336 339, 2008.

[8] C. B. Mbachu, V. E. Idigo, E. Ifeagwu, and I. I. Nsionu, "Filtration of Artifacts in ECG Signal using Rectangular Window-Based Digital Filters," International Journal of Computer Science Issues, Vol. 8, Issue 5, No. 1, 2011

[9] K. D. Chinckhede, S. Y. Govind, S. R. Hirekhan, and D. R. Solanke, "On the Implementation of FIR Filter with Various Windows for Enhancement of ECG Signal," International Journal of Engineering Science and Technology, Vol. 3, No. 3, pp. 2031 - 2040, 2011.

[10] F. J. Harris, "On the use of Windows for Harmonic Transform," Proceedings of the IEEE, Vol. 66, No. 1, pp. 51-84, 1978.

[11] R. A. Roberts and C. T. Mullis, Digital Signal Processing Reading: M. A. Addison-Wesley, 1987.

[12] J. F. Kaiser, "Non Recursive Digital Filter Design Using the Sine Window Function," Proceedings of IEEE Symposium, Circuits and System, pp. 20-23, 1974

[13] C. B. Mbachu, G. N. Onoh, V. E. Idigo, E. N. Ifeagwu, and S. U. Nnebe, "Processing ECG Signal with Kaiser Window-Based FIR Digital Filter," International Journal of Engineering Science and Technology, Vol. 3, No. 8, Pp. 6775 - 6783, 2011.

[14] K. Mukesh, P. Rohit, S. Rohini, and K. Saurabh, "Design of Band pass Finite Impulse Response Filter Using Various Research Methods," Journal of Engineering Research and Applications, Vol. 3, Issue 5, pp. 1057-1061, 2013.

[15] K. Jyoti, Y. Pramod, Y. Shivani, and K. R. Pranay, "Designing High Pass FIR Filter by Using Hamming and Hanning Window," International Journal of Scientific and Research in Engineering and Technology, Vol. 5, Issue 8, pp. 448-451, 2016.

[16] M. T. Afolabi and C. B. Mbachu, "Windowed Adaptive Filtering for Reducing Noise in Audio Signals During Transmission to Remote Locations," International Journal of Innovative Research and Development, Vol. 8, Issue 6, pp. 265-271, June 2019.

[17] C. S. Okafor and C. B. Mbachu, "Enhancing Voice Signal Processing Using Blackman-Harris Windowed Filtering Technique," American Journal of Engineering Research, Vol. 7, Issue 11, pp. 27-32, 2018.

[18] C. B Mbachu, S. A. Akaneme, and P. E. Okpagu, "Improving Speech Signal Quality in a Noisy Environment Using FIR Filter Designed with Kaiser Window," IOSR Journal of Electrical and Electronics Engineering, Vol. 14, Issue 4 Ser. II, pp. 15-29, August 2019

[19] O. S Ojo and C. B. Mbachu, "Enhancing Electrocardiographic Signal Processing Using Sine-Windowed Filtering Technique," American Journal of Engineering Research, Vol. 7, Issue 3, pp. 56-62, 2018.

[20] V. O Mmeremikwu, C. B. Mbachu, and J. P. Iloh, "A Finite Impulse Response (FIR) Filtering Technique for Enhancement of Electroencephalographic (EEG) Signal," IOSR Journal of Electrical and Electronics Engineering, Vol. 12, Issue 4 Ser. I, pp. 29-35, 2017.

[21] V. O Mmeremikwu, C. B. Mbachu, and J. P. Iloh, "Improving ECG Signal Using Nuttall Window-Based FIR Filter," International Journal of Engineering Research and Applications, Vol. 2, Issue 5, pp. 17-22, Nov. 2017 